\title{
Forced swim test induces divergent global transcriptomic alterations in the hippocampus of high versus low novelty-seeker rats
}

Pothitos M Pitychoutis ${ }^{1 * \dagger}$, Despina Sanoudou ${ }^{2 \dagger}$, Margarita Papandreou ${ }^{2}$, Dimitris Nasias ${ }^{2}$, Marianna Kouskou ${ }^{2}$, Craig R Tomlinson ${ }^{3,4}$, Panagiotis A Tsonis ${ }^{1 \dagger}$ and Zeta Papadopoulou-Daifoti ${ }^{2+}$

\begin{abstract}
Background: Many neuropsychiatric disorders, including stress-related mood disorders, are complex multi-parametric syndromes. Susceptibility to stress and depression is individually different. The best animal model of individual differences that can be used to study the neurobiology of affect regards spontaneous reactions to novelty. Experimentally, when naive rats are exposed to the stress of a novel environment, they display a highly variable exploratory activity and are classified as high or low responders (HR or $L R$, respectively). Importantly, HR and LR rats do not seem to exhibit a substantial differentiation in relation to their 'depressive-like' status in the forced swim test (FST), a widely used animal model of 'behavioral despair'. In the present study, we investigated whether FST exposure would be accompanied by phenotype-dependent differences in hippocampal gene expression in HR and LR rats.

Results: HR and LR rats present a distinct behavioral pattern in the pre-test session but develop comparable depressive-like status in the second FST session. At $24 \mathrm{~h}$ following the second FST session, HR and LR rats (stressed and unstressed controls) were sacrificed and hippocampal samples were independently analyzed on whole rat genome Illumina arrays. Functional analysis into pathways and networks was performed using Ingenuity Pathway Analysis (IPA) software. Notably, hippocampal gene expression signatures between HR and LR rats were markedly divergent, despite their comparable depressive-like status in the FST. These molecular differences are reflected in both the extent of transcriptional remodeling (number of significantly changed genes) and the types of molecular pathways affected following FST exposure. A markedly higher number of genes (i.e., 2.28-fold) were statistically significantly changed following FST in LR rats, as compared to their HR counterparts. Notably, genes associated with neurogenesis and synaptic plasticity were induced in the hippocampus of LR rats in response to FST, whereas in HR rats, FST induced pathways directly or indirectly associated with induction of apoptotic mechanisms.
\end{abstract}

Conclusions: The markedly divergent gene expression signatures exposed herein support the notion that the hippocampus of HR and LR rats undergoes distinct transcriptional remodeling in response to the same stress regimen, thus yielding a different FST-related 'endophenotype', despite the seemingly similar depressive-like phenotype.

Keywords: Stress, Gene expression, Rats, Depression, Individual differences, Hippocampus, FST, Neurogenesis, Neuroplasticity, Apoptosis

\footnotetext{
*Correspondence: ppitychoutis1@udayton.edu

${ }^{+}$Equal contributors

'Department of Biology \& Center for Tissue Regeneration and Engineering

(TREND), University of Dayton, 300 College Park, Dayton, OH 45469-2320,

USA

Full list of author information is available at the end of the article
}

\section{Biomed Central}

(c) 2014 Pitychoutis et al.; licensee BioMed Central Ltd. This is an Open Access article distributed under the terms of the Creative Commons Attribution License (http://creativecommons.org/licenses/by/2.0), which permits unrestricted use, distribution, and reproduction in any medium, provided the original work is properly credited. The Creative Commons Public Domain Dedication waiver (http://creativecommons.org/publicdomain/zero/1.0/) applies to the data made available in this article, unless otherwise stated. 


\section{Background}

Many neuropsychiatric disorders, including stress-related mood disorders, are complex multi-parametric syndromes. Strikingly, susceptibility to depression and stress differs between individuals. Accurate diagnosis is hard to establish and current pharmacotherapeutic strategies suffer from significant variability in effectiveness, making the understanding of inter-individual variations crucial to unveiling effective new treatments [1].

The best animal model of individual differences that can be used to study the neurobiology of affect regards spontaneous reactions to novelty [2]. Experimentally, when naive rats are exposed to the stress of a novel environment, they display a highly variable exploratory activity; some rats are characterized by high rates of locomotor reactivity (high responders (HR)) whereas others by low rates (low responders (LR)) (for a detailed review on the HR/LR model, the reader is referred to [2,3]). Novelty-seeking is an affect-related trait that has been associated with anxiety and emotional reactivity [4] as well as with depressive symptomatology $[5,6]$ in humans and in rodents.

The forced swim test (FST) [7] is a widely implemented animal model of behavioral despair with high predictive validity for agents with antidepressive potential [8,9]. Increased passive behavioral responses in FST, such as immobility, and decreased active behaviors like swimming or climbing are thought to be a clear indication of 'depressive-like' symptomatology [10,11]. Interestingly, male HR and LR rats do not exhibit a substantial differentiation in relation to their depressive-like status in the FST $[4,12,13]$. However, previous results from our lab indicate that HR and LR rats may exhibit a different behavioral pattern characterized by distinct active behavioral reactions expressed during either the first or the second exposure to water [4]. Moreover, we recently reported that chronic antidepressant treatment with clomipramine may attenuate depressive-like symptomatology in FST in male HR but not in their LR counterparts [12]. This evidence combined suggests that idiosyncratic responses to novelty may lead to a phenotypically similar depressivelike outcome, through different, yet unknown, molecular mechanisms.

In search of the brain structures likely to be involved in these processes, the hippocampus stands out due to its central role in the detection of novelty and the guidance of behavioral responses on the basis of familiarity [14]. Furthermore, the hippocampus has been the focus of multiple studies on the pathophysiology and treatment of stress-related disorders [15-17]. Indeed, stress often has detrimental effects on the integrity and function of the hippocampus. These effects include alterations in the activity of monoaminergic systems, in dendritic and synaptic remodeling, as well as in levels of adult hippocampal neurogenesis [18].
In the present study, we investigated whether FST exposure would be accompanied by phenotype-dependent differences in hippocampal gene expression in $\mathrm{HR}$ and LR rats. The markedly divergent gene expression signatures exposed herein support the notion that the hippocampus of HR and LR rats undergoes distinct transcriptional remodeling in response to the same stress regimen, thus yielding a different FST-related 'endophenotype', despite the seemingly similar depressivelike phenotype.

\section{Results}

HR and LR rats present with distinct behavioral pattern in the pre-test session but comparable depressive-like status in the second FST session

A one-way analysis of variance (ANOVA) showed that HR rats are characterized by higher vertical activity as compared to their LR counterparts $\left(F_{(1,23)}=36.234 ; p<0.001\right.$; Figure 1a). This level of significance was maintained when these initial groups were dichotomized in order for the control and FST groups to be formed (data not shown). Most importantly, during the pre-test FST session, a one-way ANOVA showed that LR rats spent less time swimming around the cylinder, as compared to their HR counterparts $\left(F_{(1,13)}=4.957 ; p=0.046\right.$; Figure $\left.1 \mathrm{~b}\right)$. In LR rats, this decrease in swimming was attributed to a non-significant increase of immobility. The same analysis showed that the structure of HR and LR rats' behavior was similar during the second FST session (Figure 1c). Specifically, both HR and LR rats exhibited increased floating behavior and decreased active behaviors (i.e., climbing and swimming) during the second session, which reflect the establishment of depressive-like behavior in this test.

\section{Hippocampal gene expression signature changes in HR and LR rats following exposure to FST}

The global gene expression signatures in the hippocampus of HR and LR rats were assessed in rats exposed to FST versus their respective unstressed counterparts. Bioinformatic analysis revealed significant changes in 258 transcripts (243 upregulated; 15 downregulated) in HR rats upon FST exposure (Additional files 1 and 2). On the other hand, in LR rats, 589 transcripts were statistically significantly changed, all of which were upregulated. Despite the large number of significant changes, only 20 transcripts were affected in both novelty-seeking phenotypes (Figure 2; Additional file 3).

\section{Gene Ontology (GO) classification}

To determine the biological processes and molecular functions likely to be affected by the significant gene expression changes, a comparative analysis tool provided by the 'GeneCodis3' software was used for the Gene Ontology 

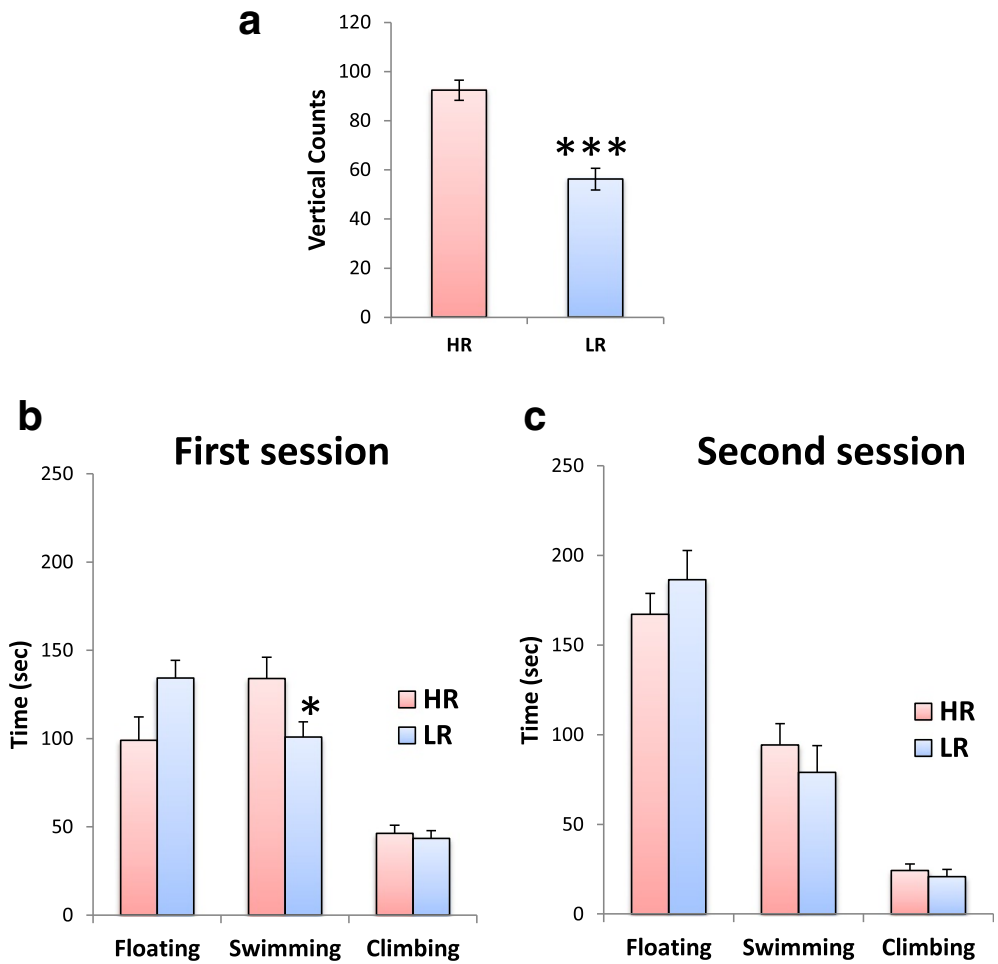

Figure 1 Behavioral data. (a) HR/LR classification: the initial classification of rats into HR or LR phenotype on the basis of their vertical locomotor activity (i.e., vertical counts) in an open field ( $N=12$ per phenotype) was assessed with one-way ANOVAs. The structure of HR and LR rats' behavior (i.e., immobility, swimming, climbing) ( $N=7$ per group) in the (b) first (pre-test) and (c) second (test) FST sessions was assessed with separate one-way ANOVAs. Bars represent means \pm SEM; ${ }^{*} p<0.05 ;{ }^{* * *} p<0.001$.

classification of the differentially expressed genes in both groups. GO level 5 annotations were obtained for 117 unique genes in HR rats and 354 unique genes in LR rats (only 9 genes were common in both groups). Focusing on the 'biological process' (BP) of the unique genes, 50 processes (annotations) in HR and 189 processes in LR rats were found to be statistically significantly changed. In HR rats, the BPs most highly affected were the 'cellular macromolecule metabolic process' (56 genes) and the 'cellular biosynthetic process' (42 genes) whereas in LR rats, the 'regulation of cellular process' (154 genes) and 'cellular macromolecule metabolic process' (125 genes). As far as the 9 common genes are concerned, no annotations were significantly enriched.

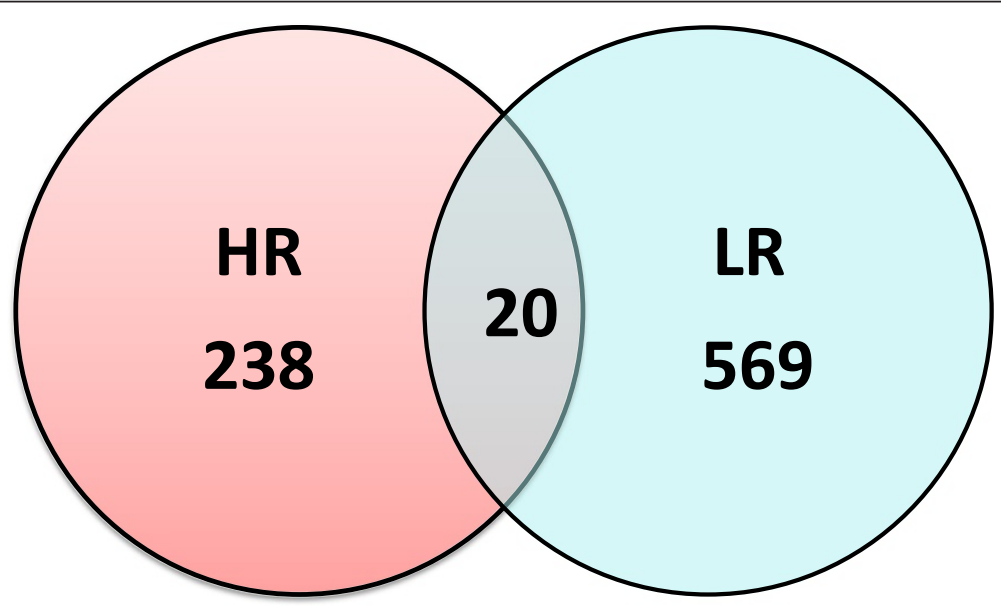

Figure 2 Exposure to FST affected a different number of transcripts in HR versus LR rats (238 versus 569). Notably, only 20 common transcripts were affected in both novelty-seeking phenotypes. 
In order to depict those processes orchestrating possible hippocampal structural and/or functional alterations in response to FST in the two novelty-seeking phenotypes, an additional set of selection criteria was applied, depicting statistically significant GO categories selected by one or more of the following keywords: neuro-, synaptic, stress, plasticity, cell death, cytoskeleton, migration, and adhesion (Figure 3). This approach unveiled even greater differences between the molecular response of HR and LR rats to FST exposure. Notably, 'synaptic transmission' (21 genes), 'cell migration' (15 genes), 'cell-cell adhesion' (10 genes), 'neurotransmitter transport' (8 genes), and 'neuroblast proliferation' (4 genes) are among the GO BPs found to be represented only in the LR group, while 'regulation of cell death' (11 HR versus 21 LR genes), 'cellular response to stress' (11 HR versus 20 LR genes), and 'actin cytoskeleton organization' (5 HR versus 11 LR genes) were found to be affected in both groups.

\section{Ingenuity Pathway Analysis (IPA)}

The GO findings were confirmed and further enriched through IPA. In HR rats, IPA analysis indicated that the observed gene expression changes were associated with 73 statistically significant functional categories including 'cellular growth and proliferation' (45 genes), 'cellular development' (24 genes), 'cellular movement' (9 genes), 'cell morphology' (19 genes), and 'nervous system development and function' (11 genes). Similarly, 76 IPA functions were affected in LR rats with 'cellular assembly and organization' (73 genes), 'cellular function and maintenance' (77 genes), 'cell-to-cell signaling and interaction' (53 genes), 'nervous system development and function' (83 genes), 'cell morphology' (74 genes), and 'cellular development' (30 genes) being among the functions overrepresented. It is noteworthy that both the GO and IPA analyses confirmed that FST exposure affected the transcription of genes related to hippocampal cellular proliferation in both noveltyseeking phenotypes.

Zooming in the specific molecular pathways associated with the observed gene expression changes, data mining through IPA exposed markedly divergent mechanisms in the hippocampus of the two novelty-seeking phenotypes in response to FST stress. Overall, 49 canonical IPA

a regulation of cell death
(positive and negative regulation of cell death) cellular response to stress actin cytoskeleton organization neurohypophysis development

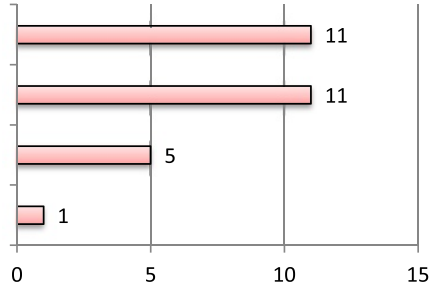

b

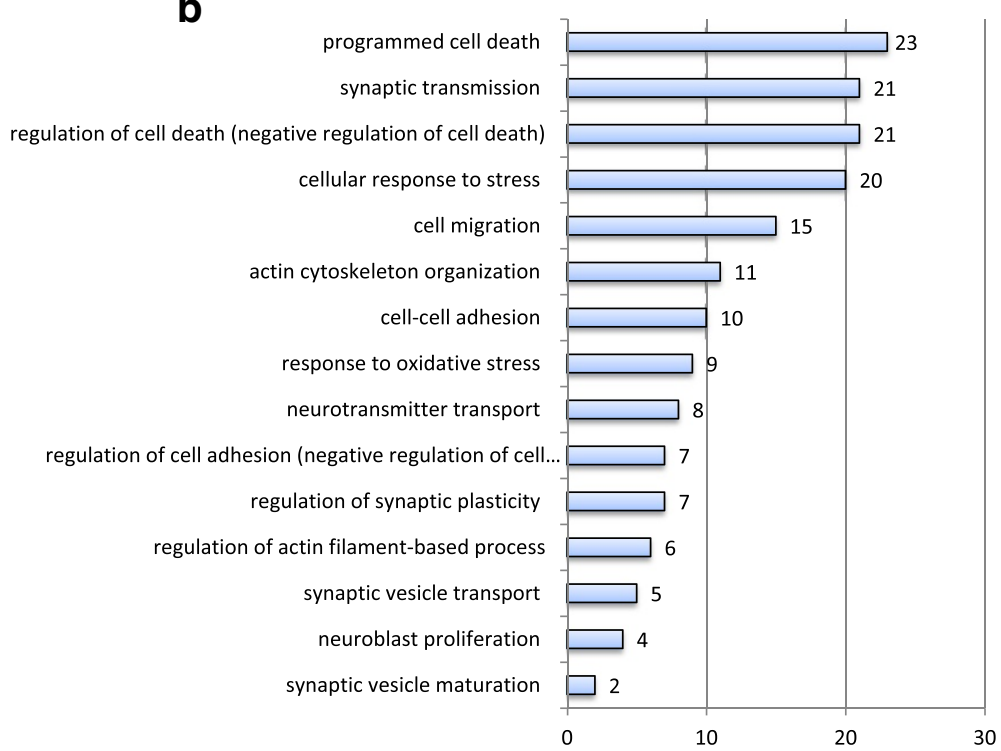

Figure 3 Distribution of differentially expressed genes according to GO BP in (a) HR and (b) LR rats. GeneCodis functional GO BP level 5 categories that meet conservative statistical criteria (hypergeometric distribution and false discovery rate (FDR) test, $p<0.05$ ) and include any one of the selected relevant keywords (i.e., neuro-, synaptic, stress, plasticity, death, cytoskeleton, migration, and adhesion). Numbers in the chart denote the number of genes grouped in each annotation. 
pathways comprising 1 to 11 genes each were depicted as significantly changed following FST in HR rats, versus 62 pathways comprising 1 to 16 genes each in LR rats. Only 15 pathways were common in both HR and LR rats, and these were largely represented by different genes due to the low number of common genes affected in both novelty-seeking phenotypes. Notably, in HR rats, 'EIF2 Signaling' and 'Axonal Guidance Signaling' were among the top five pathways affected upon FST exposure, whereas in LR rats, these were 'Ephrin Receptor Signaling', 'Clathrin-mediated Endocytosis Signaling', and 'ERK/MAPK Signaling' (Additional file 4).

\section{Mammalian Adult Neurogenesis Gene Ontology (MANGO) analysis}

In order to decipher the impact of FST exposure on the regulation of neurogenesis-related mechanisms, the MANGO was implemented [19]. In particular, the MANGO database was screened for genes that were statistically significantly changed in our data. According to this form of analysis, FST exposure induced a phenotypedependent expression of neurogenesis-related transcripts in the hippocampus of $\mathrm{HR}$ and LR rats. In particular, seven neurogenesis-related genes were statistically significantly upregulated in LR (i.e., Ephb2, Nog, Ntf3, Tgfb1, Smad7, Sox2, and Srr), as compared to only three genes in HR rats (i.e., Bmprla, Gsk3b, and Jag1). This analysis revealed that exposure to the same FST regimen induced a phenotype-dependent regulation of unique neurogenesisrelated genes in the hippocampus of the two noveltyseeking phenotypes.

\section{IPA network analysis}

Finally, network analysis was performed to unravel the intricate relationships between the different significantly changed pathways. In HR rats, 13 networks were generated by IPA versus 25 in LR rats. Consistently with all other levels of analysis, the molecular networks that emerged for the HR and LR responses to FST exposure were markedly different. This indicates that not only the individual genes and pathways are characteristic of each of the two behavioral phenotypes, but also the cascades of molecular events (i.e., the networks of genes from different pathways) giving rise to the depressive-like phenotype are distinct. The top networks of genes whose expression was significantly altered in the hippocampus of the two novelty-seeking phenotypes are presented in Figure 4.

\section{Discussion}

Herein, we report for the first time the marked divergence of the FST-induced hippocampal gene expression signatures between HR and LR rats, despite their comparable depressive-like status during the FST. These molecular differences are reflected in both the extent of transcriptional remodeling (number of significantly changed genes) and the types of molecular pathways affected following FST exposure. A markedly higher number of genes (i.e., 2.28-fold) were statistically significantly changed following FST in LR as compared to their HR counterparts (i.e., 589 versus 258), while only a strikingly low number of genes (i.e., 20 genes) were commonly altered in both novelty-seeking phenotypes. These distinct gene expression signatures support the notion that the hippocampus of $\mathrm{HR}$ and LR rats undergoes distinct transcriptional remodeling in response to the same environmentally induced stress, thus yielding a different FST-related endophenotype, despite the seemingly similar depressive-like phenotype in this test.

\section{Structure of HR and LR rats' behavior in the FST}

HR and LR rats present a distinct behavioral pattern in the pre-test session but develop comparable depressivelike status in the second FST session. Notably, LR rats spent less time swimming around the cylinder during the pre-test session, as compared to their HR counterparts. Present behavioral findings essentially replicate previous results from our lab; Antoniou et al. [4] reported that HR and LR rats develop a different behavioral FST pattern characterized by a common behavioral state of 'despair', but distinct active behavioral reactions are expressed during the first exposure to water [4]. The structure of HR and LR rats' behavior during the second FST session is similar between the two novelty-seeking phenotypes, as shown herein and as previously reported $[4,12,13]$. The majority of studies in this field typically quantify only the second FST session, as it is relevant to the screening of antidepressant drugs [12]. However, it is noteworthy that the pre-test session reflects rats' responsiveness to the presentation of a novel swim stressor that may ultimately lead to the modulation of behavior during subsequent exposure to the stressful stimulus (e.g., induction of learned helplessness) or even to the differential regulation of relevant endophenotypes (i.e., neurobiological indices) without apparent effect in the behaviors assessed [12].

\section{FST induces differential regulation of neurogenesis- related transcripts in HR compared to LR}

In the present study, both the GO and IPA analyses demonstrate that FST exposure affected the transcription of genes related to hippocampal cellular proliferation and homeostasis in both novelty-seeking phenotypes. According to the MANGO analysis, FST exposure induced a phenotype-dependent expression of neurogenesis-related genes in the hippocampus of HR and LR rats. In particular, only three neurogenesis-related genes were statistically significantly upregulated in HR (i.e., Bmprla, Gsk3b, and 


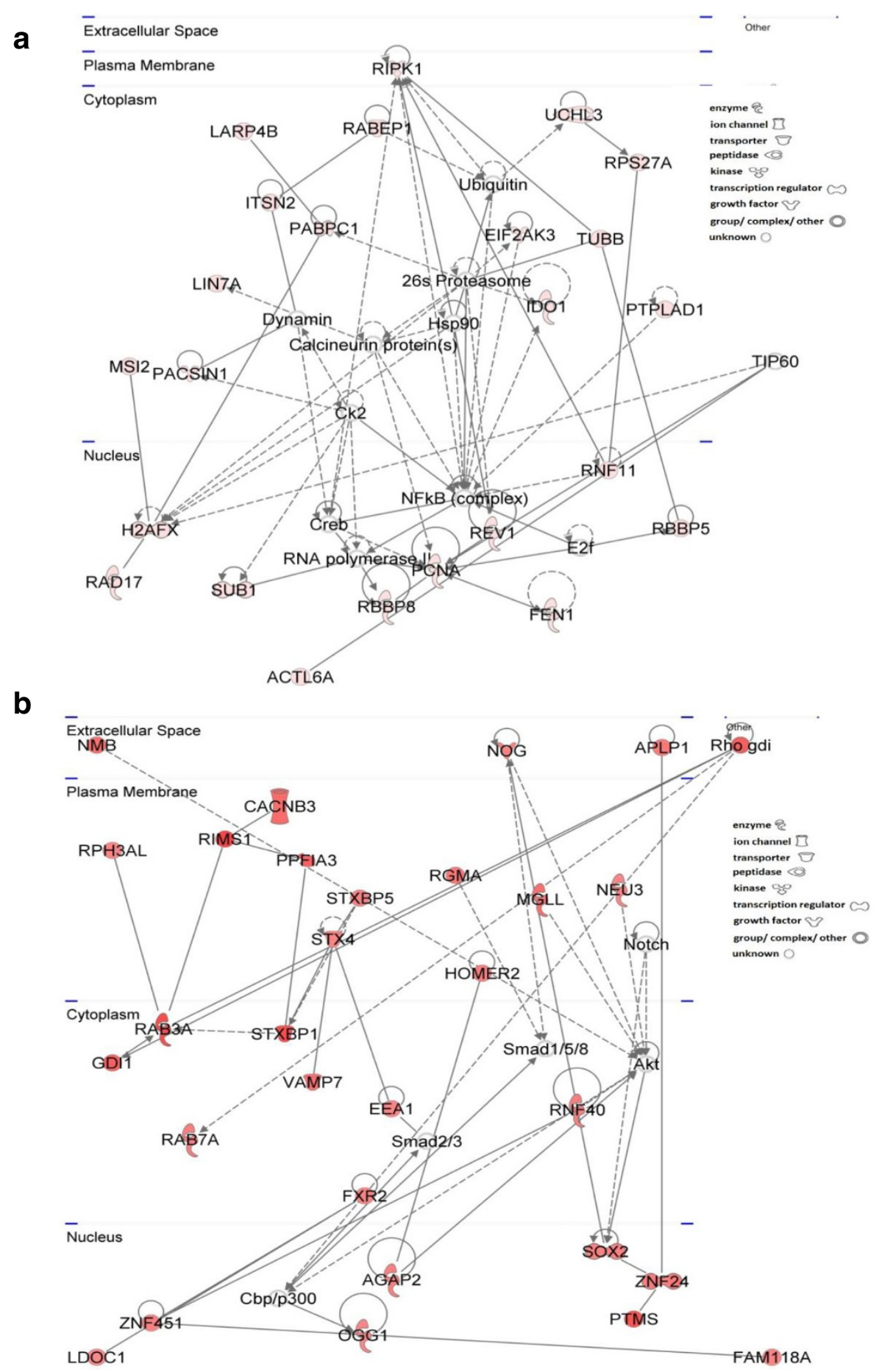

Figure 4 Top Ingenuity networks of genes whose expression was significantly altered in the hippocampus upon FST exposure. (a) HR (24 upregulated transcripts) and (b) LR (30 upregulated transcripts) rats. Upregulated molecules are shown in red and increasing color intensity reflects higher fold changes. Genes with baseline expression are shown in white. Arrows point to the activated molecule in a signaling pathway or the product of a metabolic pathway. Solid lines indicate direct and dashed lines indirect interactions. Geometrical shapes (nodes) reflect the key functions of the specific protein. 
Jag1), as compared to seven genes in LR rats (i.e., Ephb2, Nog, Ntf3, Tgfb1, Smad7, Sox2, and Srr). Importantly, exposure of HR rats to the FST paradigm induced hippocampal Gsk3b (glycogen synthase kinase 3 beta; +2.089) mRNA levels. Gsk3b is a negative regulator of the b-catenin and the canonical Wnt signaling pathway, which modulate hippocampal neurogenesis [20,21], but has also been reported to modulate Notch signaling by enhancing its stability [22]. Notably, overexpression of Gsk3b in the murine hippocampus causes dramatic alterations in both the dendritic tree morphology and the postsynaptic densities of newborn neurons and has been proposed as a target for the treatment of Alzheimer's disease [23]. Moreover, FST exposure in HR rats upregulated Bmprla (bone morphogenetic protein receptor, type IA; +2.275) and Jag1 (jagged; +2.163; the ligand of Notch1), which have been reported to affect neurogenesis in the adult hippocampus either directly or indirectly $[24,25]$.

In LR rats, the FST induced the transcriptional activation of genes that positively regulate neurogenesis in the adult hippocampus. Notably, Ntf3 (neurotrophin $3 ;+2.469$ ) facilitates hippocampal plasticity by promoting neurogenesis in the dentate gyrus [26], Nog (noggin; +2.177) is a BMP antagonist that diverts stem cells from a glial to a neuronal fate [27], and Sox2 (SRY (sex-determining region Y)-box 2 ; +2.201) maintains neural stem cell properties, including proliferation/survival, self-renewal, and neurogenesis [28]. Moreover, FST exposure led to overexpression of $T g f b 1$ (transforming growth factor, beta $1 ;+2.219)$ and Smad7 (SMAD family member $7 ;+2.443$ ) mRNA in the hippocampus of LR rats. Interestingly, TGFb1 has been recognized as a negative regulator of adult neurogenesis since infusion of TGFb1 into the ventricles of the adult rat brain reduced the number of proliferating cells in the hippocampus [29], while SMAD7 may either directly antagonize TGF receptor signaling [30] or act in a TGF receptor-independent manner and further suppress neurogenesis [31]. Although not registered in the MANGO database, several fibroblast growth factor (FGF)-related genes were upregulated in the hippocampus of LR rats following FST, namely Fgf12 (fibroblast growth factor 12; +3.116), Fgf13 (fibroblast growth factor $13 ;+2.465$ ), Fgfr2 (fibroblast growth factor receptor 2 ; +2.088 ), and Frs3 (fibroblast growth factor receptor substrate $3 ;+2.113$ ). Intriguingly, upregulation of Fgfr2 upon FST exposure may mediate the neuroprotective effects of FGF2 on hippocampal formation [32]. Thus, it could be hypothesized that FST has a beneficial effect in the hippocampus of LR rats by promoting neuroprotective processes.

Intriguingly, FST induced phenotype-dependent effects on the transcription of prominent serotonin (5-HT)related enzymes. In the hippocampus of LR rats, FST upregulated Tph2 (tryptophan hydroxylase 2 ; +2.145), the rate-limiting enzyme of 5 -HT biosynthesis, that is possibly indicative of serotonergic activation in this brain region. On the other hand, in HR rats, Ido1 (indoleamine 2,3-dioxygenase; +2.302) levels were induced. It is noteworthy that IDO1 is a proinflammatory enzyme that converts L-tryptophan (the precursor of serotonin; $5-\mathrm{HT}$ ) to kynurenine and its neurotoxic metabolites that are able to produce oxidative stress by increasing the production of reactive oxygen species (ROS) or to overstimulate hippocampal $N$-methyl-D-aspartate (NMDA) receptors and lead to apoptosis and hippocampal atrophy [33].

Overall, FST exposure induced a phenotype-dependent modulation of neurogenesis-related genes in the hippocampus of the two novelty-seeking phenotypes. Notably, the hippocampus is a stress- and glucocorticoid (GC)sensitive brain region. In particular, within the dentate gyrus (DG), there exists a high density of glucocorticoid receptors (GRs) that respond to enhanced circulating GCs [34]. According to previous reports from our group and others, serum corticosterone concentrations are enhanced by FST exposure irrespective of the HR/LR phenotype $[12,13]$ and this could negatively affect hippocampal cell proliferation [35]. Moreover, it has been shown that hippocampal DNA damage occurs immediately after exposure of male Wistar rats to FST [36] and that exposure of female rats to the same regimen may reduce cell survival in the hippocampus, probably by increasing corticosterone levels [37]. Indeed, this profile nicely fits in with the transcriptomic remodeling observed in HR rats in response to FST in the current study. On the other hand, recent data also support a beneficial role for acute stress on the hippocampus. In particular, exposure of rats to restraint stress increased cell proliferation and astrocytic fibroblast growth factor 2 ( $f g f 2)$ expression in the dorsal hippocampus [38]. Remarkably, in the present study, exposure of LR rats to FST resulted in enhanced expression of hippocampal Ffgr2 mRNA levels. Importantly, FST appears to promote neurogenic processes in LR rats. These findings are in accordance with the enhanced neurogenesis observed in the DG of LR rats at baseline. As shown before, cell proliferation in the DG of LR Wistar rats, evaluated by the incorporation of 5-bromo-2' -deoxyuridine (Brdu) in progenitors, was twice that observed in their HR counterparts [39]. Similarly, selectively bred HR and LR (bHR/bLR) lines of Sprague-Dawley rats revealed, through Ki67 immunohistochemistry, enhanced cell proliferation in the DG of developing bLR versus bHR pups [40]. In conclusion, the increased rates of hippocampal neurogenesis in LR rats at baseline appear to be maintained in response to FST-induced stress, with the activation of numerous relevant processes. These molecular changes contribute to the markedly different molecular milieu of LR compared to HR rats that may predispose to distinct long-term behavioral or pharmacological responses. 


\section{FST induced upregulation of neuroplasticity-related transcripts in LR rats}

Neuroplasticity refers to the brain's ability to change its structure and function during maturation, learning, environmental challenges, pathology, and stress. Synaptic pruning and remodeling of the postsynaptic cytoskeleton comprise structural changes that are possible in the adult brain. Interestingly, exposure to FST upregulated genes directly or indirectly involved in neuroplasticity in a phenotype-specific manner in LR rats. Ephrins and their receptors govern the topographic guidance of axons during central nervous system (CNS) development but are also implicated in neuronal plasticity in the adult brain [41]. Herein, the "Ephrin Receptor Signaling" pathway was the second among the top five canonical IPA pathways affected in the hippocampus of LR rats upon FST exposure (Figure 5). Specifically, FST resulted in the upregulation of both Ephb2 (+2.167) and Epha5 (+2.879) transcripts. Ephrin receptors are the largest known family of receptor tyrosine kinases in the mammalian genome and are divided into $A$ and $B$ subclasses based on affinity for their membrane-associated ligands, ephrin-As and ephrin-Bs. Notably, Eph $\alpha 5$ is expressed at both the protein and
mRNA levels in the adult murine hippocampus where it has been hypothesized to promote synaptic plasticity [42]. Most importantly, Ephb2 is expressed in the adult hippocampus and regulates, among others, synaptic structure, NMDA receptor clustering and function, and LTP [43].

In line with this evidence, the IPA functions predicted to be statistically significantly enhanced in LR rats upon FST exposure involved 'growth of neurites' ( $z$-score 2.825), 'LTP of synapse' ( $z$-score 2.396), 'microtubule dynamics' ( $z$-score 2.453), 'organization of cytoplasm' ( $z$-score 2.276), and 'organization of cytoskeleton' ( $z$-score 2.269), all of which are directly related with neuroplasticity. Of particular interest was the upregulation of a number of neuroplasticity-related synaptic players in the hippocampus of LR rats, such as $D d n$ (dendrin; +2.225 ), a postsynaptic regulator of the structure of synaptic cytoskeleton, and Stx4 (syntaxin $4 ;+2.178$ ), an essential postsynaptic component for synaptic plasticity in hippocampal neurons [44]. Moreover, the upregulation of both $P f n$ (profilin) isoforms (i.e., 1 and 2; +2.709 and 2.396, respectively) that are prominent regulators of actin dynamics in the CNS is indicative of the cytoskeletal reorganization that possibly takes place upon FST exposure in the

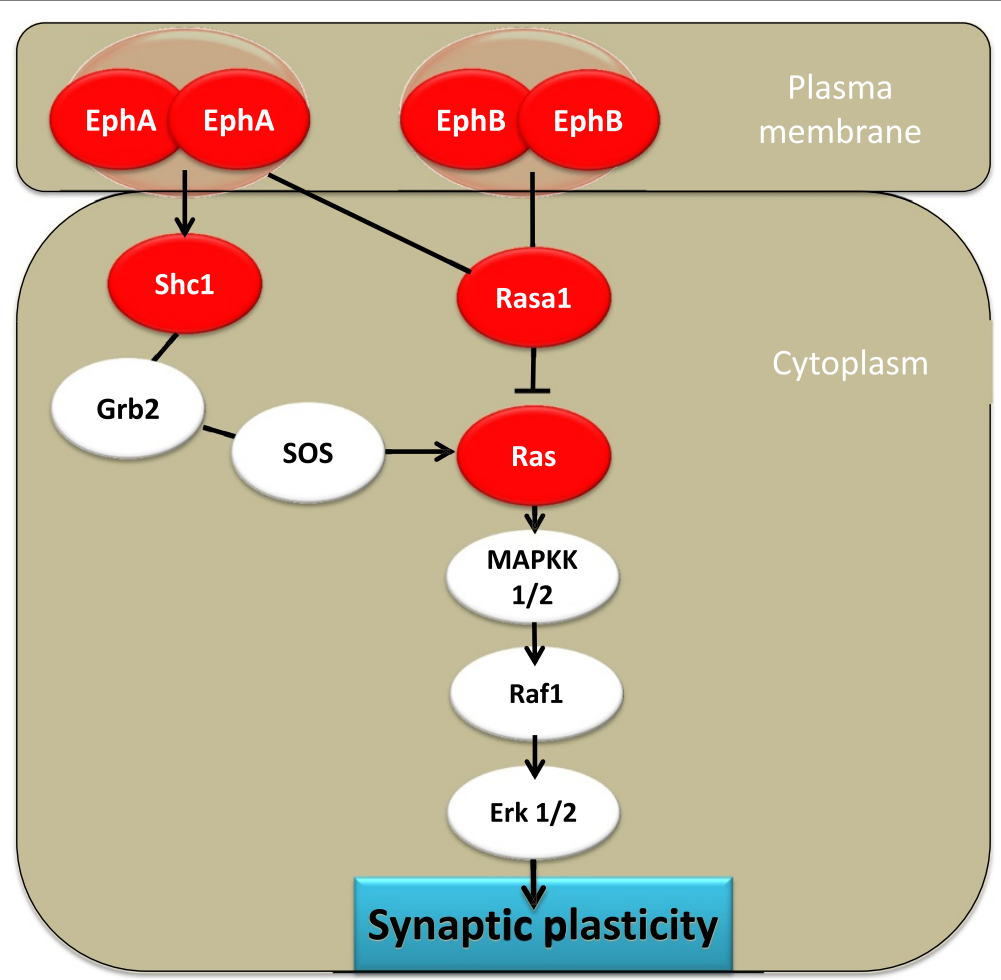

Figure 5 'Ephrin Receptor Signaling' was the second canonical IPA pathway induced in LR rats upon FST exposure. Upregulated molecules are shown in red. Blind lines denote binding, arrows signify activation, whereas T-like lines indicate inhibition. EphA5 Ephrin A5 receptor, EphB2 Ephrin B2 receptor, Erk1/2 mitogen-activated protein kinase 1, GRB2 growth factor receptor-bound protein 2, MAPKK1/2 mitogen-activated protein kinase 2, RASA1 RAS p21 protein activator (GTPase-activating protein) 1, RAF1 v-raf-1 murine leukemia viral oncogene homolog 1, Shc1 Src homology 2 domain containing) transforming protein 1, SOS son of sevenless homolog 2. 
hippocampus of LR rats [45]. Moreover, FST appears to stimulate neurotransmitters' release in LR rats, as reflected by the upregulation of Dnm1 (dynamin 1; +2.388 ) which is involved in exo-endocytosis of synaptic vesicles [46] and Syt12 (synaptotagmin 12; +2.352 ) which is a synaptic vesicle phosphoprotein that modulates neurotransmitter release [47]. Hippocampal synaptotagmin mRNA levels were found to be enhanced upon immobilization stress in rats, in view of the effects of stress on synaptic plasticity [48]. Remarkably, exposure of LR rats to FST resulted in the upregulation of genes implicated in core neuroadaptive processes in the adult hippocampus.

\section{FST induced apoptosis-related mechanisms in the hippocampus of HR rats}

The top canonical IPA pathway affected in HR rats upon FST exposure was that of eIF2 (eukaryotic translation initiation factor 2) signaling. The eIF2 complex is essential in all eukaryotes for protein synthesis, since it recruits the initiator methionyl tRNA (Met-tRNA) to ribosomes to begin translation. In addition, multiple endoplasmic reticulum (ER) stress-related genes were overexpressed in the hippocampus of HR rats following FST exposure, including Perk (eif2ak3; eIF2a kinase 3; +2.053), Sgpp2 (sphingosine-1-phosphate (S1P) phosphatase 2; +3.121), and Mbtps2 (membrane-bound transcription factor peptidase $2 ;+2.593)$. As depicted in Figure 6, Perk upregulation could lead to phosphorylation-induced inhibition of eIF $2 \alpha$ that could in turn result in global reduction of protein translation. In addition, upregulation of $G s k 3 b(+2.089)$ may inhibit the activity of eIF2b (eukaryotic initiation factor $2 \mathrm{~B}$ ) that could also lead to inhibition of protein translation in neuronal cells [49]. Interestingly, it has been proposed that inhibition of protein translation is a mode of inducing neuronal apoptosis and neurodegeneration in Alzheimer's disease [50,51]. Notably, induction of Mbtps 2 that encodes an intramembrane zinc metalloprotease involved in ER stress response may enhance the activation of target genes related to the ER stress pathway through an Atf-6 (activating transcription factor 6)-dependent mechanism. In addition, Sgpp2 is an enzyme localized in cell and ER membranes that forms sphingosine from S1P that in turn is N-acylated to ceramide; both ceramide and sphingosine have been associated with induction of ER stress, growth arrest, and apoptosis in mammalian cells [52-54]. FST also upregulated Rip1 (TNF receptor (TNFRSF)-interacting serinethreonine kinase $1 ;+2.249)$, a kinase originally discovered via its interaction with the death domain of TNFR1 [55]. Importantly, Rip1 is a central initiator of cell death and may also play a role in the ceramide death pathway [51]. Overall, it appears that in HR rats FST induced the overexpression of multiple genes directly and

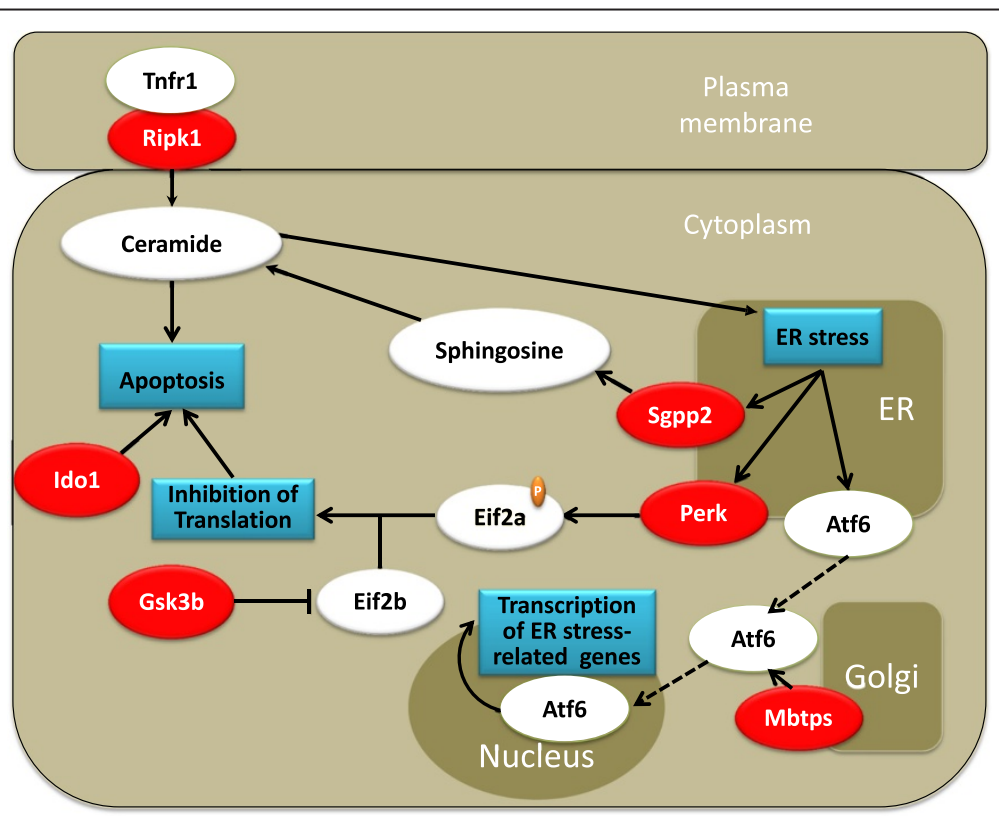

Figure 6 ER stress and elF2a pathways appear to be induced in the hippocampus of HR rats. Induction of these pathways could be implicated in the mobilization of apoptotic mechanisms in hippocampal neurons of HR rats upon FST exposure. Upregulated genes are shown in red. Blind lines denote binding, arrows signify activation, whereas T-like lines indicate inhibition. ATF-6 activation transcription factor 6, elF2a eukaryotic initiation factor 2a, elF2b eukaryotic initiation factor 2b, Gsk3b glycogen synthase kinase-3 beta, Ido 1 indoleamino-2,3-dioxygenase, MBTPS membrane-bound transcription factor peptidase, Perk elF2a kinase 3, Sgpp2 sphingosine-1-phosphate (S1P) phosphatase 2, Ripk1 TNF receptor (TNFRSF)-interacting serine-threonine kinase 1, TNFR1 tumor necrosis factor receptor superfamily. 
indirectly associated with apoptotic mechanisms in the adult hippocampus.

\section{Conclusions}

Herein, we report that FST-induced hippocampal gene expression signatures between HR and LR rats are markedly divergent. The two novelty-seeking phenotypes present a distinct behavioral pattern in the pre-test session but develop comparable depressive-like status in the second session of the FST. At $24 \mathrm{~h}$ post-FST exposure, hippocampal gene expression signatures between HR and LR rats were markedly divergent, despite their similar FST performance. These molecular differences are reflected in both the extent of transcriptional remodeling (i.e., number of significantly changed genes) and the types of molecular pathways affected following FST exposure. A markedly higher number of genes (i.e., 2.28-fold) were statistically significantly changed following FST in LR rats, as compared to their HR counterparts. Specifically, global transcriptomic alterations suggest that following FST exposure, neurogenesis and neuroplasticity-related processes are enhanced in LR rats, while pathways that are directly or indirectly implicated in apoptotic cascades are induced in their HR counterparts. Importantly, the present data extend previous findings on the differential modulation of neurogenesis between the two novelty-seeking phenotypes at baseline.

Overall, the present data suggest a complex pattern of molecular modifications that likely reflect distinct neurobiological processes occurring between the adult HR and LR hippocampus upon FST exposure. Strikingly, susceptibility to stress is individually different. Gaining an insight into individual differences may elucidate the neurobiological substrate for this vulnerability, help clarify pathophysiological mechanisms, and hopefully aid in developing strategies to discover more effective pharmacotherapies [2].

\section{Methods}

\section{Animals}

Male Sprague-Dawley rats $(N=24)$ were purchased from Athens' Pasteur Institute. Rats aged 80-90 days old and weighed 250-300 $\mathrm{g}$ at the beginning of the experiments. Rats were group housed in plastic cages measuring $570 \mathrm{~mm} \times 380 \mathrm{~mm} \times 200 \mathrm{~mm}$, with food pellets and tap water available ad libitum, under controlled laboratory conditions (i.e., 12-h light/dark with lights on at 0700 hours and a constant temperature of $21^{\circ} \mathrm{C} \pm 1^{\circ} \mathrm{C}$ ), as previously described [12]. Approval to conduct the experiments described has been obtained from the animal subjects review board of our institution. All animal experiments have been carried out in accordance with the European Communities Council Directive of 24 November
1986 (86/609/EEC). Efforts were made to minimize the numbers of animals used and to reduce their suffering.

\section{Classification into HR/LR groups}

All rats were classified as HR and LR according to their frequency of rearing (counts registered when the rat's body inclined vertically with hind paws on the floor and forepaws on the wall of the cage) in an activity chamber, as in previous studies $[4,12,56,57]$. Vertical activity has been used, apart from general locomotion, as a sole criterion for assignment of rats into groups during their exposure to novelty $[58,59]$. The rats were ranked using the frequency of vertical counts (rearing). Animals above the median were designated as $\mathrm{HR}$, while the rest were classified as LR. Testing was conducted in two clear Plexiglas activity chambers measuring $430 \times 430 \times 300 \mathrm{~mm}$ (Med Associates Inc., St Albans, VT, USA), and behavior was recorded for a 15-min observation period, as previously described [12,56].

\section{Forced swim test (FST)}

All rats were acclimatized to the test room for $1 \mathrm{~h}$ before the beginning of the experiment. HR and LR rats $(N=7$ per phenotype) were individually placed in a cylindrical tank measuring $50 \mathrm{~cm}$ in height and $20 \mathrm{~cm}$ in width or were left undisturbed in their home cage and served as controls ( $N=5$ per phenotype). The tank was filled with water $\left(24^{\circ} \mathrm{C} \pm 1^{\circ} \mathrm{C}\right)$, and water was changed after each session. The animals were forced to swim for a 15-min period (pre-test) and $24 \mathrm{~h}$ later were subjected to a 5-min swimming session (test) [60]. The total duration of floating (immobility), swimming, and climbing periods were scored by the same observer for the first $5 \mathrm{~min}$ of each session (pre-test and test), as mentioned before $[12,61]$. Rats were considered to show immobility when they floated without struggling, making only those movements necessary to keep their heads above the water. Swimming was recorded when they actively swam around in circles, while climbing was scored when the rats climbed the walls of the cylinder. Following swimming sessions, the rats were removed from the tank, carefully dried in heated cages, and then returned to their home cages. At $24 \mathrm{~h}$ following the second session of the FST, all HR and LR rats (stressed and respective controls) were sacrificed by rapid decapitation with the aid of a guillotine. Notably, in earlier studies, we and others have chosen this wait period in order to examine prolonged stress-induced effects on gene expression alterations within a short time period following the stress session $[17,61-63]$.

\section{RNA isolation and qualitative and quantitative analyses}

Following decapitation, the brain was removed from the skull and the region of the hippocampus was rapidly isolated on ice. Sample preparation, hybridization, washing, 
and staining were performed using the direct hybridization gene expression assay by Illumina, involving a first- and second-strand reverse transcription step, followed by a single in vitro transcription (IVT) amplification that incorporates biotin-labeled nucleotides, array hybridization, washing, blocking, and streptavadin-Cy3 staining. Hippocampal samples ( $N=5$ per group per phenotype) were analyzed independently on whole rat genome Illumina arrays (RatRef-12 Expression BeadChip), containing 22,523 probes selected primarily from the NCBI RefSeq database (Release 16), representing approximately 22,260 coding transcripts. The arrays were scanned using the BeadArray Reader (Illumina, San Diego, CA, USA).

\section{Statistics, bioinformatic analysis, and data mining}

Statistical analysis of behavioral data was performed with SPSS version 20 statistical software (SPSS Inc., Chicago, IL, USA). Results were analyzed using one-way analysis of variance (ANOVA) for the factor of phenotype (HR versus LR). Specifically, separate one-way ANOVAs were performed for each FST session. All data were first tested against ANOVA data assumptions.

The resulting gene expression data sets from the 20 hippocampal samples were scanned using the Illumina GenomeStudio Gene Expression Module (version 2010.2) at default specifications and thresholds. The scanned microarray image files were pre-processed using quantile normalization without background correction [64]. Bioinformatic analysis was performed using the BRB-Array Tools Version 4.2.1 [65]. All intensity values were transformed to the $\log 2$ basis. Differentially expressed genes were then identified using a random-variance $t$ test, with a $p$ value threshold of $\leq 0.01$. To increase stringency, only genes with $\geq 2$-fold change were further considered for the purposes of this study. Significantly changed genes were annotated according to the Gene Ontology classification system into 'biological process' (BP), 'molecular function' (MF), and 'cellular component' (CC) by using the GeneCodis3 software, a web tool for functional interpretation of experimental data. The application is freely available at http://genecodis.cnb.csic.es [66-68]. In particular, unique gene symbols (ENTREZ) corresponding to HR and LR data sets, respectively, were processed in the application 'Comparative analysis' of GeneCodis3 for Rattus norvegicus, the GO level (level 5), and the statistical parameters of the analysis. The submission of two different lists at the same time results in simultaneous modular and singular enrichment analyses for each one. Hypergeometric distribution was used for the calculation of $p$ values and the FDR method to correct $p$ values for multiple hypothesis testing. Statistical significance for both tests was determined at the $p<0.05$ level. Annotations were then retrieved for each data set in order for unique and common genes to be further analyzed.
Literature mining and biological interpretation of significantly changed genes was performed with the Ingenuity Pathway Analysis software (IPA, Ingenuity Systems, www.ingenuity.com). Data of analysis are experimentally observed and come from Ingenuity Knowledge Base. Fisher's exact test is used for the grouping of analyzed genes into biofunctions and canonical pathways by calculating the $p$ value of a given category. Statistical significance association of genes to the functional or pathway categories is considered for $p$ values less than 0.05 and is expressed as the negative log of the $p$ value. Additionally, the activation state of a functional category is predicted by calculating the $z$-score. A predicted increase in activation of a function is considered when the $z$-score is $\geq 2$, and accordingly, a predicted decrease is considered when the $z$-score is $\leq 2$.

In order to decipher the impact of FST exposure on the regulation of neurogenesis-related mechanisms, the Mammalian Adult Neurogenesis Gene Ontology (MANGO) was further implemented [19]. The MANGO accounts for a database of genes mapped to cell types and processes that have been curated from the literature concerning adult neurogenesis [19]. Herein, the MANGO database was screened for genes that were statistically significantly changed in our data sets.

\section{Additional files}

Additional file 1: HR datasheet. Statistically significantly changed transcripts between FST-exposed and control (unstressed) HR rats.

Additional file 2: LR datasheet. Statistically significantly changed transcripts between FST-exposed and control (unstressed) LR rats.

Additional file 3: Common transcripts between HR and LR.

Statistically significantly changed transcripts that were similarly affected in $H R$ and LR rats in response to FST.

Additional file 4: IPA pathways. Top-5 of statistically significantly changed IPA pathways in HR and LR rats in response to FST.

\section{Competing interests}

The authors declare that they have no competing interests.

\section{Authors' contributions}

PMP, DS, PAT, and ZPD conceived the study and participated in its design and coordination, the interpretation of results, and the drafting of the manuscript. MP, DN, and MK performed the bioinfomatic analysis of the microarray data. CRT participated in the generation of the microarray data. All authors read and approved the final draft.

\section{Acknowledgements}

This work was supported by grants from the Special Account for Research Grants of the University of Athens (Greece) and from the National Institutes of Health grant EY16707. The input of Dr. R. Cowper-Sal-lari in the initial stages of the microarray analysis is greatly appreciated. The funders had no role in the study design, data collection and analysis, decision to publish, or preparation of the manuscript.

\section{Author details}

${ }^{1}$ Department of Biology \& Center for Tissue Regeneration and Engineering (TREND), University of Dayton, 300 College Park, Dayton, OH 45469-2320, USA. ${ }^{2}$ Department of Pharmacology, Medical School, National \& Kapodistrian 
University of Athens, 75 Mikras Asias StreetGoudi, Athens 11527, Greece. ${ }^{3}$ Department of Medicine, Dartmouth Hitchcock Medical Center, Dartmouth

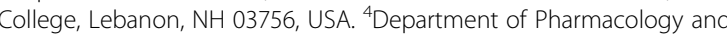
Toxicology, Dartmouth Hitchcock Medical Center, Dartmouth College, Lebanon, NH 03756, USA.

Received: 27 November 2013 Accepted: 14 February 2014 Published: 25 February 2014

\section{References}

1. Duclot F, Hollis F, Darcy MJ, Kabbaj M: Individual differences in noveltyseeking behavior in rats as a model for psychosocial stress-related mood disorders. Physiol Behav 2011, 104:296-305.

2. Harro J: Inter-individual differences in neurobiology as vulnerability factors for affective disorders: implications for psychopharmacology. Pharmacol Ther 2010, 125:402-422.

3. Blanchard MM, Mendelsohn D, Stamp JA: The HR/LR model: further evidence as an animal model of sensation seeking. Neurosci Biobehav Rev 2009, 33:1145-1154.

4. Antoniou K, Papathanasiou G, Papalexi E, Hyphantis T, Nomikos GG, Spyraki C, Papadopoulou-Daifoti Z: Individual responses to novelty are associated with differences in behavioral and neurochemical profiles. Behav Brain Res 2008, 187:462-472

5. Hansenne M, Reggers J, Pinto E, Kjiri K, Ajamier A, Ansseau M: Temperament and character inventory (TCl) and depression. J Psychiatr Res 1999, 33:31-36.

6. Hur JW, Kim YK: Comparison of clinical features and personality dimensions between patients with major depressive disorder and normal control. Psychiatry Investig 2009, 6:150-155.

7. Porsolt RD, Anton G, Blavet N, Jalfre M: Behavioural despair in rats: a new model sensitive to antidepressant treatments. Eur J Pharmacol 1978, 47:379-391.

8. Borsini F: Role of the serotonergic system in the forced swimming test. Neurosci Biobehav Rev 1995, 19:377-395.

9. Cryan JF, Markou A, Lucki I: Assessing antidepressant activity in rodents: recent developments and future needs. Trends Pharmacol Sci 2002, 23:238-245

10. Detke MJ, Rickels M, Lucki I: Active behaviors in the rat forced swimming test differentially produced by serotonergic and noradrenergic antidepressants. Psychopharmacology (Berl) 1995, 121:66-72.

11. Lopez-Rubalcava C, Lucki I: Strain differences in the behavioral effects of antidepressant drugs in the rat forced swimming test. Neuropsychopharmacology 2000, 22:191-199.

12. Pitychoutis PM, Pallis EG, Mikail HG, Papadopoulou-Daifoti Z: Individual differences in novelty-seeking predict differential responses to chronic antidepressant treatment through sex- and phenotype-dependent neurochemical signatures. Behav Brain Res 2011, 223:154-168.

13. Jama A, Cecchi M, Calvo N, Watson SJ, Akil H: Inter-individual differences in novelty-seeking behavior in rats predict differential responses to desipramine in the forced swim test. Psychopharmacology 2008, 198:333-340.

14. Knight RT, Nakada T: Cortico-limbic circuits and novelty: a review of EEG and blood flow data. Rev Neurosci 1998, 9:57-70.

15. Nestler EJ, Barrot M, DiLeone RJ, Eisch AJ, Gold SJ, Monteggia LM: Neurobiology of depression. Neuron 2002, 34:13-25.

16. Pittenger C, Duman RS: Stress, depression, and neuroplasticity: a convergence of mechanisms. Neuropsychopharmacology 2008, 33:88-109.

17. Pitychoutis PM, Dalla C, Sideris AC, Tsonis PA, Papadopoulou-Daifoti Z: 5-HT (1A), 5-HT(2A), and 5-HT(2C) receptor mRNA modulation by antidepressant treatment in the chronic mild stress model of depression: sex differences exposed. Neuroscience 2012, 210:152-167.

18. Dalla C, Pitychoutis PM, Kokras N, Papadopoulou-Daifoti Z: Sex differences in response to stress and expression of depressive-like behaviours in the rat. Curr Top Behav Neurosci 2011, 8:97-118.

19. Overall RW, Paszkowski-Rogacz M, Kempermann G: The mammalian adult neurogenesis gene ontology (MANGO) provides a structural framework for published information on genes regulating adult hippocampal neurogenesis. PloS One 2012, 7:e48527.

20. Hur EM, Zhou FQ: GSK3 signalling in neural development. Nat Rev Neurosci 2010, 11:539-551.

21. Guo W, Murthy AC, Zhang L, Johnson EB, Schaller EG, Allan AM, Zhao X Inhibition of GSK3beta improves hippocampus-dependent learning and rescues neurogenesis in a mouse model of fragile $X$ syndrome. Hum Mol Genet 2012, 21:681-691.

22. Foltz DR, Santiago MC, Berechid BE, Nye JS: Glycogen synthase kinase-3beta modulates notch signaling and stability. Curr Biol 2002, 12:1006-1011.

23. Llorens-Martin M, Fuster-Matanzo A, Teixeira CM, Jurado-Arjona J, Ulloa F, Defelipe J, Rabano A, Hernandez F, Soriano E, Avila J: GSK-3beta overexpression causes reversible alterations on postsynaptic densities and dendritic morphology of hippocampal granule neurons in vivo. Mol Psychiatry 2013, 18:451-460

24. Mira H, Andreu Z, Suh H, Lie DC, Jessberger S, Consiglio A, San Emeterio J, Hortiguela R, Marques-Torrejon MA, Nakashima K, Colak D, Götz M, Fariñas I, Gage FH: Signaling through BMPR-IA regulates quiescence and long-term activity of neural stem cells in the adult hippocampus. Cell Stem Cell 2010, 7:78-89.

25. Ables $J$, Breunig JJ, Eisch AJ, Rakic P: Not(ch) just development: Notch signalling in the adult brain. Nat Rev Neurosci 2011, 12:269-283.

26. Shimazu K, Zhao M, Sakata K, Akbarian S, Bates B, Jaenisch R, Lu B: NT-3 facilitates hippocampal plasticity and learning and memory by regulating neurogenesis. Learn Mem 2006, 13:307-315.

27. Bonaguidi MA, Peng CY, McGuire T, Falciglia G, Gobeske KT, Czeisler C, Kessler JA: Noggin expands neural stem cells in the adult hippocampus. J Neurosci 2008, 28:9194-9204.

28. Favaro R, Valotta M, Ferri AL, Latorre E, Mariani J, Giachino C, Lancini C Tosetti V, Ottolenghi S, Taylor V, Nicolis SK: Hippocampal development and neural stem cell maintenance require Sox2-dependent regulation of Shh. Nat Neurosci 2009, 12:1248-1256.

29. Wachs FP, Winner B, Couillard-Despres S, Schiller T, Aigner R, Winkler J, Bogdahn $U$, Aigner L: Transforming growth factor-beta1 is a negative modulator of adult neurogenesis. J Neuropathol Exp Neurol 2006, 65:358-370.

30. Kandasamy M, Reilmann R, Winkler J, Bogdahn U, Aigner L: Transforming growth factor-beta signaling in the neural stem cell niche: a therapeutic target for Huntington's disease. Neurol Res Int 2011, 2011:124256.

31. Krampert M, Chirasani SR, Wachs FP, Aigner R, Bogdahn U, Yingling JM, Heldin $\mathrm{CH}$, Aigner L, Heuchel R: Smad7 regulates the adult neural stem/ progenitor cell pool in a transforming growth factor beta- and bone morphogenetic protein-independent manner. Mol Cell Biol 2010 30:3685-3694

32. Chadashvili T, Peterson DA: Cytoarchitecture of fibroblast growth factor receptor 2 (FGFR-2) immunoreactivity in astrocytes of neurogenic and non-neurogenic regions of the young adult and aged rat brain. J Comp Neurol 2006, 498:1-15

33. Wichers MC, Maes M: The role of indoleamine 2,3-dioxygenase (IDO) in the pathophysiology of interferon-alpha-induced depression. J Psychiatry Neurosci 2004, 29:11-17.

34. Sousa N, Almeida OF: Corticosteroids: sculptors of the hippocampal formation. Rev Neurosci 2002, 13:59-84.

35. Cameron HA, Tanapat P, Gould E: Adrenal steroids and N-methyl-D aspartate receptor activation regulate neurogenesis in the dentate gyrus of adult rats through a common pathway. Neurosci 1998, 82:349-354

36. Consiglio AR, Ramos AL, Henriques JA, Picada JN: DNA brain damage after stress in rats. Prog Neuropsychopharmacol Biol Psychiatry 2010, 34:652-656

37. Vega-Rivera NM, Fernandez-Guasti A, Ramirez-Rodriguez G, EstradaCamarena E: Acute stress further decreases the effect of ovariectomy on immobility behavior and hippocampal cell survival in rats. Psychoneuroendocrinology 2013, 38:1407-1417.

38. Kirby ED, Muroy SE, Sun WG, Covarrubias D, Leong MJ, Barchas LA Covarrubias D, Leong MJ, Barchas LA, Kaufer D: Acute stress enhances adult rat hippocampal neurogenesis and activation of newborn neurons via secreted astrocytic FGF2. elife 2013, 2:e00362.

39. Lemaire V, Aurousseau C, Le Moal M, Abrous DN: Behavioural trait of reactivity to novelty is related to hippocampal neurogenesis. Eur J Neurosci 1999, 11:4006-4014.

40. Clinton SM, Stead JD, Miller S, Watson SJ, Akil H: Developmental underpinnings of differences in rodent novelty-seeking and emotional reactivity. Eur J Neurosci 2011, 34:994-1005.

41. Xiao D, Miller GM, Jassen A, Westmoreland SV, Pauley D, Madras BK: Ephrin/Eph receptor expression in brain of adult nonhuman primates: implications for neuroadaptation. Brain Res 2006, 1067:67-77. 
42. Cooper MA, Crockett DP, Nowakowski RS, Gale NW, Zhou R: Distribution of EphA5 receptor protein in the developing and adult mouse nervous system. J Comp Neurol 2009, 514:310-328.

43. Henderson JT, Georgiou J, Jia Z, Robertson J, Elowe S, Roder JC, Pawson T: The receptor tyrosine kinase EphB2 regulates NMDA-dependent synaptic function. Neuron 2001, 32:1041-1056.

44. Mohanasundaram $P$, Shanmugam MM: Role of syntaxin 4 in activitydependent exocytosis and synaptic plasticity in hippocampal neurons. Sci Signal 2010, 3:jc7.

45. Murk K, Wittenmayer N, Michaelsen-Preusse K, Dresbach T, Schoenenberger CA, Korte M, Jockusch BM, Rothkegel M: Neuronal profilin isoforms are addressed by different signalling pathways. PLoS One 2012, 7:e34167.

46. Mallozzi C, D'Amore C, Camerini S, Macchia G, Crescenzi M, Petrucci TC, Di Stasi AM: Phosphorylation and nitration of tyrosine residues affect functional properties of Synaptophysin and Dynamin I, two proteins involved in exo-endocytosis of synaptic vesicles. Biochimica Et Biophysica Acta 2013, 1833:110-121.

47. Maximov A, Shin OH, Liu X, Sudhof TC: Synaptotagmin-12, a synaptic vesicle phosphoprotein that modulates spontaneous neurotransmitter release. J Cell Biol 2007, 176:113-124.

48. Thome J, Pesold B, Baader M, Hu M, Gewirtz JC, Duman RS, Henn FA: Stress differentially regulates synaptophysin and synaptotagmin expression in hippocampus. Biol Psychiatry 2001, 50:809-812.

49. Webb BL, Proud CG: Eukaryotic initiation factor 2B (elF2B). Int J Biochem Cell Biol 1997, 29:1127-1131.

50. Chang RC, Wong AK, Ng HK, Hugon J: Phosphorylation of eukaryotic initiation factor-2alpha (elF2alpha) is associated with neuronal degeneration in Alzheimer's disease. Neuroreport 2002, 13:2429-2432.

51. Thon L, Mohlig H, Mathieu S, Lange A, Bulanova E, Winoto-Morbach S, Schutze S, Bulfone-Paus S, Adam D: Ceramide mediates caspaseindependent programmed cell death. FASEB J 1945-1956, 2005:19

52. Spiegel S, Milstien S: Sphingosine-1-phosphate: an enigmatic signalling lipid. Nat Rev Mol Cell Biol 2003, 4:397-407.

53. Mandala SM: Sphingosine-1-phosphate phosphatases. Prostaglandins Other Lipid Mediat 2001, 64:143-156.

54. Chen CL, Lin CF, Chang WT, Huang WC, Teng CF, Lin YS: Ceramide induces p38 MAPK and JNK activation through a mechanism involving a thioredoxininteracting protein-mediated pathway. Blood 2008, 111:4365-4374.

55. Stanger BZ, Leder P, Lee TH, Kim E, Seed B: RIP: a novel protein containing a death domain that interacts with Fas/APO-1 (CD95) in yeast and causes cell death. Cell 1995, 81:513-523.

56. Polissidis A, Chouliara O, Galanopoulos A, Rentesi G, Dosi M, Hyphantis T, Marselos M, Papadopoulou-Daifoti Z, Nomikos GG, Spyraki C, Tzavara ET, Antoniou K: Individual differences in the effects of cannabinoids on motor activity, dopaminergic activity and DARPP-32 phosphorylation in distinct regions of the brain. Int J Neuropsychopharmacol 2010, 13:1175-1191.

57. Topic B, Dere E, Schulz D, de Souza Silva MA, Jocham G, Kart E, Huston JP: Aged and adult rats compared in acquisition and extinction of escape from the water maze: focus on individual differences. Behav Neurosci 2005, 119:127-144.

58. Antoniou K, Papathanasiou G, Panagis G, Nomikos GG, Hyphantis T, Papadopoulou-Daifoti Z: Individual responses to novelty predict qualitative differences in d-amphetamine-induced open field but not reward-related behaviors in rats. Neurosci 2004, 123:613-623.

59. Thiel CM, Muller CP, Huston JP, Schwarting RK: High versus low reactivity to a novel environment: behavioural, pharmacological and neurochemical assessments. Neurosci 1999, 93:243-251.

60. Porsolt RD, Le Pichon M, Jalfre M: Depression: a new animal model sensitive to antidepressant treatments. Nature 1977, 266:730-732.

61. Drossopoulou G, Antoniou K, Kitraki E, Papathanasiou G, Papalexi E, Dalla C, Papadopoulou-Daifoti Z: Sex differences in behavioral, neurochemical and neuroendocrine effects induced by the forced swim test in rats. Neuroscience 2004, 126:849-857.

62. Lopez JF, Liberzon I, Vazquez DM, Young EA, Watson SJ: Serotonin 1A receptor messenger RNA regulation in the hippocampus after acute stress. Biol Psychiatry 1999, 45:934-937.

63. Shishkina GT, Kalinina TS, Berezova IV, Bulygina W, Dygalo NN: Resistance to the development of stress-induced behavioral despair in the forced swim test associated with elevated hippocampal Bcl-xl expression. Behav Brain Res 2010, 213:218-224.
64. Wright GW, Simon RM: A random variance model for detection of differential gene expression in small microarray experiments. Bioinformatics 2003, 19:2448-2455.

65. Smyth GK, Speed T: Normalization of CDNA microarray data. Methods 2003, 31:265-273.

66. Tabas-Madrid D, Nogales-Cadenas R, Pascual-Montano A: GeneCodis3: a non-redundant and modular enrichment analysis tool for functional genomics. Nucleic Acids Res 2012, 40:W478-W483.

67. Nogales-Cadenas R, Carmona-Saez P, Vazquez M, Vicente C, Yang X, Tirado F, Carazo JM, Pascual-Montano A: GeneCodis: interpreting gene lists through enrichment analysis and integration of diverse biological information. Nucleic Acids Res 2009, 37:W317-W322.

68. Carmona-Saez P, Chagoyen M, Tirado F, Carazo JM, Pascual-Montano A: GENECODIS: a web-based tool for finding significant concurrent annotations in gene lists. Genome Biol 2007, 8:R3.

doi:10.1186/1479-7364-8-4

Cite this article as: Pitychoutis et al:: Forced swim test induces divergent global transcriptomic alterations in the hippocampus of high versus low novelty-seeker rats. Human Genomics 2014 8:4.

\section{Submit your next manuscript to BioMed Central and take full advantage of:}

- Convenient online submission

- Thorough peer review

- No space constraints or color figure charges

- Immediate publication on acceptance

- Inclusion in PubMed, CAS, Scopus and Google Scholar

- Research which is freely available for redistribution

Submit your manuscript at www.biomedcentral.com/submit
C Biomed Central 